\title{
MICROEVOLUTION IN MIOCENE BRIZALINA (FORAMINIFERA) STUDIED BY CANONICAL VARIATE ANALYSIS AND ANALYSIS OF LANDMARKS
}

\author{
- Fred L. Bookstein \\ Center for Human Growth and Development, \\ University of Michigan, \\ Ann Arbor, Michigan, U.S.A. \\ - Richard A. Reyment \\ Paleontologiska Institutionen, \\ Uppsala Universitet, Box 558, \\ S-751 22 Uppsala, Sweden
}

\begin{abstract}
A stratigraphically oriented series of the Miocene foraminiferal species Brizalina mandoroveensis from Ikang, Cameroon, was analyzed both by conventional multivariate morphometric procedures and by the tensor biometric method of Bookstein (1986; Statist. Sci. 1, 181-142), a method which analyzes sets of landmark points rather than specific variables of shape or size. The conventional analysis used five size-measures upon 170 specimens from five stratigraphic levels; the tensor analysis encompassed six landmarks (12 coordinates) upon 50 specimens. Whereas certain features appeared in both analyses, such as the separation between levels one and five, the techniques did not always agree with respect to the interpretation of those findings or about most details in the sequence of mean phenotypes. The canonical variate analysis bases its ordination upon a general size factor (the meaning of which is obscured by the foreshortening of within-group variation which is built into the technique). The tensor analysis locates a similar ordination using mainly features of shape.
\end{abstract}

1. Introduction. The data analyzed in this paper come from a borehole drilled in Miocene sediments at Ikang, Cameroon, West Africa by the ELFSNEAP(E) Petroleum Company, France. Among the many bolivinids in these marine sediments, the species Brizalina mandoroveensis (Graham, DeKlasz, Rérat) is particularly informative for the purpose of studying time-correlated variation in shape.

The collection derives from five sampling levels in the borehole. The youngest sample was taken from a depth of around $1716 \mathrm{~m}$, the second from around $1740 \mathrm{~m}$, the third from around $1763 \mathrm{~m}$, the fourth from around $1787 \mathrm{~m}$, and the deepest sample from around $1810 \mathrm{~m}$. The collection was kindly made available by Dr L. Brun, Head of the Biostratigraphical Laboratory of ELFSNEAP(E) (Boussens). Microspheric individuals, numbering somewhat less than $10 \%$ of the collection, were omitted from all analyses. The practically important aspect of our pilot study for the petroleum industry is that it offers a 
new means for obtaining more accurate results in quantitative palaeoecological work.

The microfossils were first studied by standard methods of multivariate morphometric analysis, in particular the methods of principal components and canonical variates. These analyses were supplemented by graphical and statistical treatments of the coordinates of landmarks measured at strategic points around the test. Five "conventional" variables were measured on the tests of the foraminifers: the length of the test, its maximum breadth, the lengths of the last two chambers, and the diameter of the megalospheric proloculus (see Fig. 1).

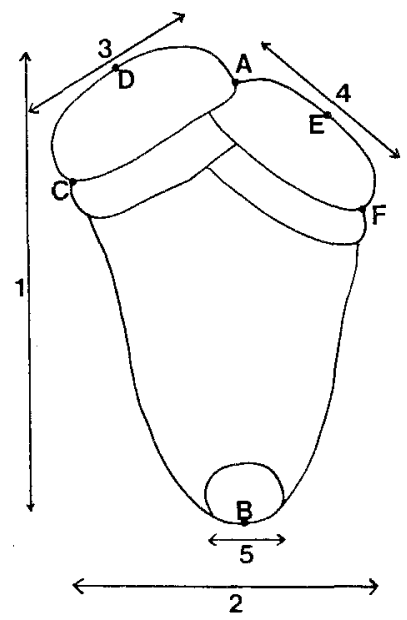

Figure 1. Measurement of Brizalina mandoroveensis. The five size-measures for the standard multivariate analyses. Landmark points for Brizalina: (A) aperture; (B) proloculus; (C) proximal boundary of the last chamber; (D) bulge of the last chamber; $(\mathrm{E})$ bulge of the second last chamber; $(\mathrm{F})$ proximal boundary of the second last chamber.

Time-correlated morphometrical studies require graphical representations of the subtle variations of shape and size that occur during the evolutionary history of a species. Conventional methods of measuring fossil shells are dominated by size-sensitive characters, so that the inclusion of shapedeterminants occurs mainly by default. It is difficult to design a standard multivariate analysis that can adequately account for evolution in shape and the manifestations of polymorphism in outline. To a certain extent, this may be managed by the various methods of "size correction" and, indirectly, by canonical variate analysis, but it is not clear how to select size measures in such a pattern that the resulting analysis will be most sensitive to the predominantly geometrical aspects of evolution in shape. This is a knotty problem for the 
Foraminifera, a group well known for the difficulties attached to the study of its variability in shape.

A way out of the difficulty is to use shape-oriented measurements instead of sizes. The most accessible approach to such measures involves the recording of the coordinates of landmarks, i.e. diagnostic points on the shell, and then applying the graphical and statistical analyses of Bookstein et al. (1985) and Bookstein (1986) directly to those data. In the following, we shall be concerned with weighing the results obtained by conventional multivariate statistical methods against those yielded by the tensor-analytical approach of Bookstein (1986).

2. Conventional Multivariate Analysis. The basic descriptive statistics for our five samples of Brizalina are displayed in Table I for each of the five measures on the text. For length, the coefficient of variation varies little over the sequence. For all the other characters, this statistic varies considerably across samples. Breadth of the test and length of the last chamber seem to show a decrease in variability over time. The remarkably high variability of the proloculi from levels 3 and 4 may indicate that the samples contain pseudomegalospheres (Nyholm, 1962).

The within-groups covariance matrix and the corresponding correlation matrix are listed in Table II. The correlations among the four dimensions of the test are all in the range of $0.60-0.85$. These values are rather high and are all statistically significant. Proloculus diameter is moderately correlated (about 0.4 ) with three of the other four characters. This may be because proloculus size influences the geometry of the way in which the subsequent chambers are constructed (cf. Reyment, 1982).

The five samples of Brizalina were analyzed by the method of canonical variate analysis in the space of the correlations (i.e. the computations were made on the pooled within-groups correlation matrix), using an expanded version of the program published in Blackith and Reyment (1971). Our results were not easily translatable to the biological problem at hand. Seemingly by way of analogy, it has become customary to interpret the coefficients of the normalized canonical vectors, or their equivalent, as if they pertained to singlesample principal components. Several examples of this attempt are cited in Reyment et al. (1984). In some analyses of non-biological materials with identically distributed samples, one arrives in this way at a useful interpretation of the coefficients of canonical variate analysis. Although some biological data are suited to this procedure, especially when the within-group covariance matrix is nearly spherical, it is not possible to proceed routinely in this respect; rather, each case must be judged on its own merits.

The canonical variate analysis for separation of our five levels is presented in Table III. Figure 2 shows the scatter of our sample in the plane of the first two 


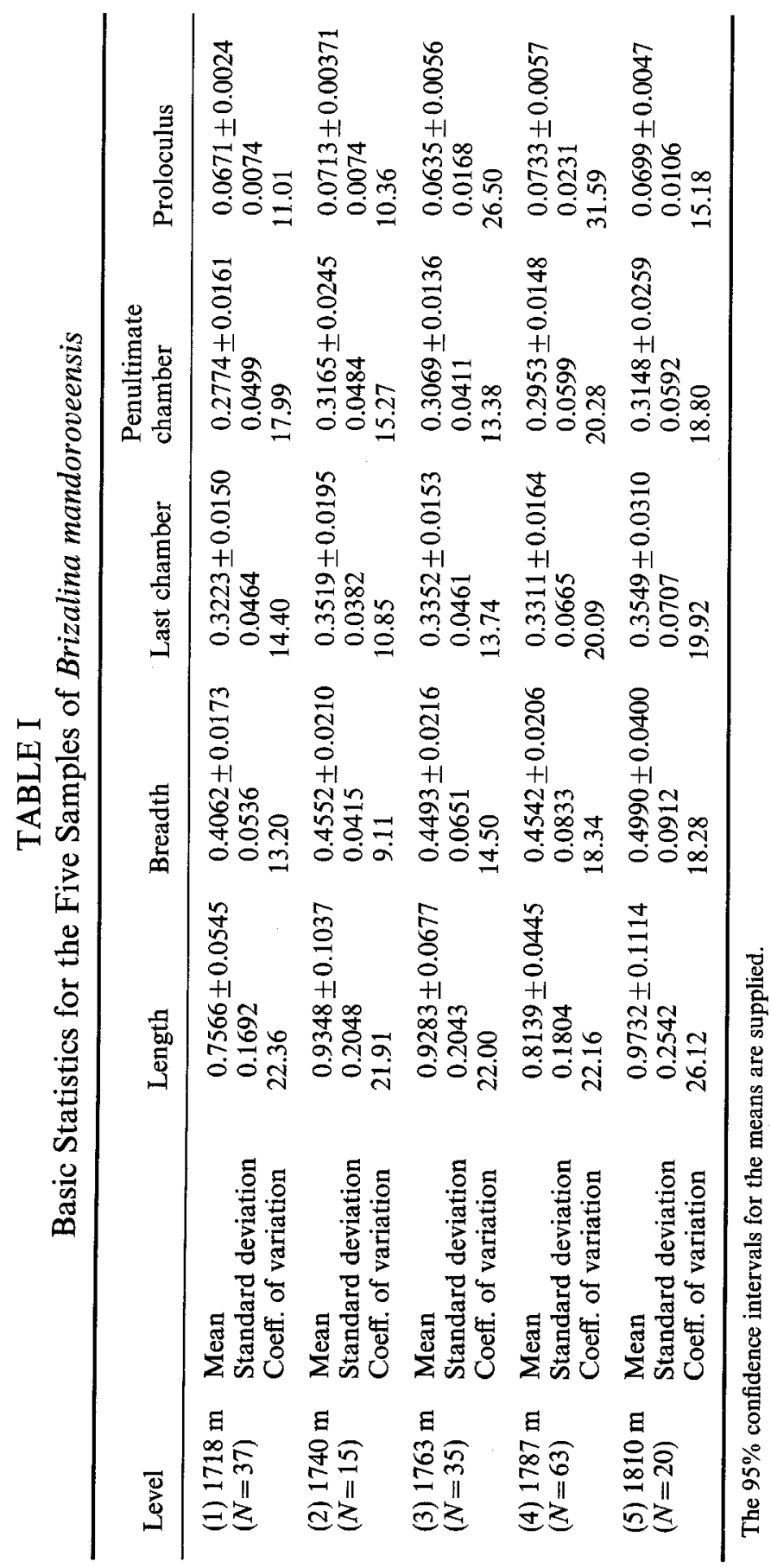


TABLE II

Within Groups Covariances and Corresponding Correlations

\begin{tabular}{cccccc}
\hline & 1 & 2 & 3 & 4 & 5 \\
1 & 6.2818 & 1.4179 & 1.1810 & 1.1068 & 0.0804 \\
2 & 1.4179 & 0.8603 & 0.5526 & 0.4681 & 0.0897 \\
3 & 1.1810 & 0.5526 & 0.5393 & 0.4246 & 0.0626 \\
4 & 1.1068 & 0.4681 & 0.4246 & 0.4686 & 0.0564 \\
5 & 0.0804 & 0.0897 & 0.0625 & 0.0564 & 0.0477 \\
& & & & & \\
& 1 & 2 & 3 & 4 & 5 \\
1 & 1.00000 & 0.60994 & 0.64165 & 0.64513 & 0.14695 \\
2 & 0.60994 & 1.00000 & 0.81125 & 0.73721 & 0.44296 \\
3 & 0.64165 & 0.81125 & 1.00000 & 0.84468 & 0.38973 \\
4 & 0.64513 & 0.73721 & 0.84468 & 1.00000 & 0.37741 \\
5 & 0.14695 & 0.44296 & 0.38973 & 0.37741 & 1.00000 \\
\hline
\end{tabular}

(1) Length of the test; (2) breadth of test; (3) length of last chamber; (4) length of second last chamber; (5) diameter of the proloculus.

canonical variates. The five sample means are indicated, and also the convex hulls of samples 1 and 5, the pair of samples found to be most distinctly separable. It appears that samples $1,2,3$, and 5 are approximately ordinated along CV1 and that sample 4 diverges from this line. (The latent roots indicate that CV's 3 and 4 are of negligible importance.) In understanding these two dimensions of principal contrast, the canonical coefficients are of surprisingly little use. In particular, the elements of the first normed latent vectorapproximately $(0.3,0.6,-0.7,0.2,-0.1)$ - do not seem to embody an interpretable pattern of covariance among the five characters, even though the separation of samples 1 and 5 is aligned with it almost perfectly. These groups tend to be opposite with respect to some morphological characteristic, but we cannot tell which.

2.1. Interpretation. Canonical variate analysis is relative eigenanalysis of two matrices, one representing between-group variation and the other withingroup variation. The latent vectors account for group separation expressed as a ratio to within-group variance. In the present application, both matrices are dominated by a single factor which can be seen by inspection to have the meaning of general size. In the within-group matrix we saw this clearly in the pattern of consistently large correlations among measures 1 through 4 . In the comparisons between levels, we notice as well a general trend of decrease in size from level 5 through level 1, most notably on variables 2 and 1 .

In the presence of such a common principal component, the interpretation of the coefficients of the canonical variates is almost impossible. The problem is 


\section{TABLE III}

Summary of the Canonical Variate Analysis for the Five Levels of Brizalina mandoroveensis $(N=170)$. Latent roots and vectors of the determinantal equation of canonical variate analysis

\begin{tabular}{lrrrr} 
& Vector 1 & Vector 2 & Vector 3 & Vector 4 \\
Length & 0.6167 & -0.7058 & 0.2431 & -0.3089 \\
Breadth & 1.1501 & 1.1746 & 0.0985 & 0.7439 \\
Last chamber & -1.1772 & -0.4383 & 1.6957 & -0.3784 \\
Second last chamber & 0.2980 & -0.3308 & -1.6698 & -0.3927 \\
Proloculus & -0.2663 & 0.4373 & -0.1084 & -0.8034 \\
Latent roots & 0.2655 & 0.1241 & 0.0195 & 0.0178 \\
The normed latent vectors & & & & \\
Length & & & & \\
Breadth & 0.3421 & -0.4584 & 0.1014 & -0.2449 \\
Last chamber & 0.6381 & 0.7629 & 0.0411 & 0.5897 \\
Second last chamber & -0.6532 & -0.2847 & 0.7075 & -0.3000 \\
Proloculus & 0.1654 & -0.2148 & -0.6967 & -0.3112 \\
& -0.1477 & 0.2840 & -0.0452 & -0.6368 \\
\hline
\end{tabular}

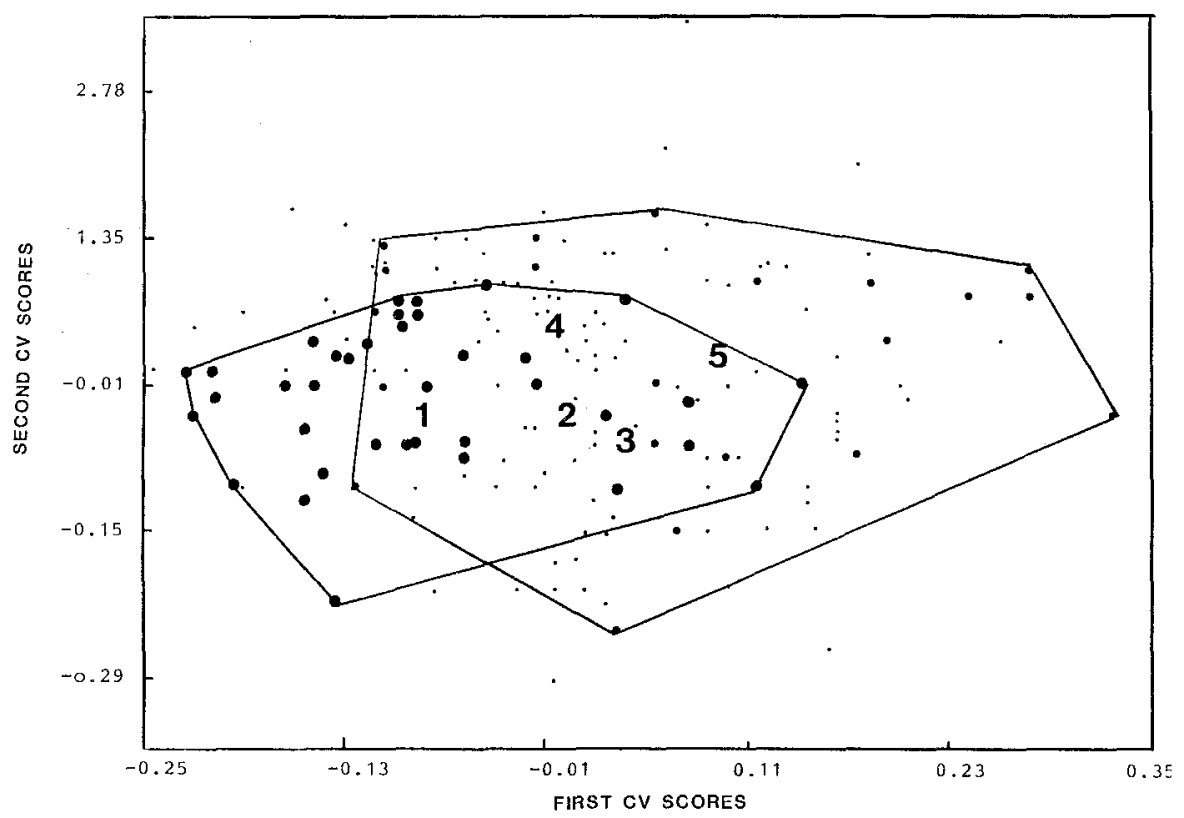

Figure 2. Scatter plot of the scores for the first two canonical variates for the set of five size variables and 170 specimens. The points for sample $1(1740 \mathrm{~m})$ are indicated by the largest dots of the diagram; the points for sample $5(1810 \mathrm{~m})$ are indicated by middle-sized dots. Points from samples 2, 3 and 4 are represented by the smallest dots. The means for the five samples are also indicated. The coefficients underlying this canonical variate representation are presented in Table III. 
the same as that induced by multicollinearity in a multiple regression or a twogroup discrimination (cf. Section 4.3 of Bookstein, 1990). To the extent that all variables are strongly positively correlated, a linear combination like $v_{1}-v_{2}$ will have little variance, and so a combination like $12 v_{1}-10 v_{2}$ will have covariances about the same as those of $v_{1}+v_{2}$ for purposes of group separation. In a suggestive geometric terminology, the first principal component of the group has been foreshortened-it is rotated away from the axis $(1,1,1,1,1)$ by which we recognize alternate measures of General Size. Recognition of these alternate measures is still possible by summing their coefficients. The sum is a very crude estimate of covariance with the sum of the measured variables, one common proxy for size.

In the present example, the eigenanalysis foreshortens the between-group size factor and scatters its coefficients, but still leaves it as a measure of size. The foreshortened coefficients for the first canonical variate total 0.60 , much more than for any of the other canonical variates. The coefficients appear to emphasize the contrast between variable 2, which decreases some $20 \%$ over the series, and variable 3 , which decreases less than $10 \%$ in spite of its larger variance within groups. An appropriate interpretation would proceed instead in terms of the canonical loadings, correlations of the measures with the variate to which they all contribute. These are positive for all of the first shapevariables, indicating that the diversity of signs of the coefficients is merely an outcome of collinearity of measures. The meaning of $C V 1$ here is General Size; only its formula is odd, owing to the specific optimization that is incorporated in the analysis.

In addition to the general size-gradient in these data, there is one other striking feature in the table of group means: the anomalous drop in variable 1 for level four. Such an effect involves roughly equal components of "size" (sum of the variables) and "shape" (here, the ratio of $V 1$ to the sum of the others). Because of this latter aspect, this comparison is not affected quite as much by the foreshortening along intragroup size brought about by the latent root extraction; indeed, it dominates $C V 2$ (cf. Fig. 2). On this $C V$, variable 2, which does not change in mean value from group 4 to group 2, is assigned a large positive coefficient, and variables 1,3 , and 4 , middling negative coefficients. This separation of variables according to the apparent shape of their timetrend-evenly upward (not found here), upward with a plateau (variable 2), or upward with a reversal (variables 1,3,4) - corresponds to their loadings on a secondary factor for timing according to the explanation of Hopkins (1966) (cf. p. 195 of Bookstein et al., 1985). Nonetheless, the pattern of coefficients on the first canonical variable cannot be interpreted in accordance with the separate patterns of group means up the series. We will return to a consideration of the canonical analysis after setting out an alternative approach to separating these same samples. 
3. Analysis of Landmarks. The five length-measures of the multivariate analysis just reviewed, like most other sets of morphometric scalars (Reyment et al., 1984), are intended as measures of size across regions which are homologous according to the usual comparative criteria. In this manner, distances measured upon forms separately may also be considered to be homologous quantities, that is, quantities that correspond from form to form. The multivariate analysis, then, becomes a study of the set of relationships among the forms of a level or the levels of a series. Each such relationship, each comparison between profiles of sample means, or each canonical variate, may be thought of as a change of form detectable by its effects upon the five distances we selected.

An alternative approach to the description of morphological change was first called to the attention of biologists by D'Arcy Thompson (1917). Thompson noted that our concept of biological homology, applied to drawings of organisms, closely resembles the geometric notion of a smooth plane mapping. For any pair of forms in a study, the homology map is a pairing of their infinitely many mathematical "points". It is as if the effect of evolution might be traced out, locus by locus, along the path bridged by the ruler in the measurement of distances.

To construe biological homology as a geometric mapping makes possible an alternate quantitative approach to the study of change in form. Instead of dealing indirectly with homology by the separate measurement of regions, one may statistically analyze the mapping function directly, by sampling it at particular loci called landmarks. Landmarks are points declared a priori to be "homologous" from form to form just as if they were embryonically or evolutionarily traceable organs or tissues. In fact, the usual method by which landmarks are operationally characterized is by reference to the structures on which they lie. A landmark might be, for instance, the point of juxtaposition of two adjacent organs or shell-structures, such as point $\mathrm{A}$ in Fig. 1, which represents the aperture of the foraminifer. In another sort of definition, a landmark might be characterized as a point within a single organ at which a certain geometric property is distinctive. For instance, our point B, "proloculus", is located where the projected curvature of the proximal edge is greatest.

\subsection{Data. From the 170 specimens of Brizalina subjected to conventional} multivariate analysis of the 5 distances, we selected 50,10 from each sampling level, upon the SEM photographs of which we could confidently locate the 6 landmarks shown in Fig. 1. These 50 specimens were not the only forms in the full data set for which the landmarks could be found, but the smallest borehole sample was of 15 specimens only, and we saw little point in oversampling the larger collections. The names, letter codes, and operational definitions of the landmarks are as follows. 
Proloculus (B). The point of sharpest curvature around the first chamber of the test, at the proximal end. This point, located by one of us (FLB) by hand, is near the proximal endpoint of the longest diameter of the form, which is measure 1, "length", in the canonical variate analysis preceding.

Proximal boundary of the final chamber (C). The point at the edge of the test at which the convexity of the final chamber appears to spring from the earlier body wall. This point is sometimes the intersection of two apparent curves on the picture and sometimes the terminus of a suture which can plainly be seen. When all three features are visible, they agree well. Point C is close to one endpoint of measure 3, "length of final chamber", in the canonical variate analysis.

Aperture (A). The point on or near the edge of the test at which the convexity of the final chamber appears to spring from that of the penultimate chamber. Point $A$ is located as the intersection of two curves. It is near the endpoint of two chamber length measures from the canonical variate analysis.

Proximal boundary of the penultimate chamber (F). The point at the edge of the test at which the convexity of the penultimate chamber appears to spring from the earlier body wall.

Bulge of the final chamber (D). The point on the edge of the final chamber at the greatest distance from a line connecting the limits of the base of this chamber, points $\mathrm{A}$ and $\mathrm{C}$, as previously located. The distance to point $D$ from this baseline corresponds to the notion of the height of this chamber. This point was located by hand with the aid of a transparent Cartesian grid. Point D is near an endpoint of measure 1, "length", in the canonical variate analysis. Note that this measure fails to meet the criterion in pp. 6-8 of Bookstein et al. (1985) for a good landmark: it is not characterized by any anatomical feature in its vicinity. We shall see the consequences of this failure as the statistical analysis unrolls.

Bulge of the penultimate chamber $(\mathrm{E})$. The point on the edge of the penultimate chamber at the greatest distance from a line connecting the limits of the base of this chamber, points $\mathrm{A}$ and $\mathrm{F}$, as previously located.

3.2. Biometric analysis of landmark locations. Over the last 20 years, several statistical methods have been developed for the analysis of two-dimensional landmark data (Sneath, 1967; Bookstein, 1978; Brower and Veinus, 1978; Bookstein, 1982; Siegel and Benson, 1982; Strauss and Bookstein, 1982; Goodall, 1983; Bookstein et al., 1985). A recent synthesis of these methods has proved suitable for routine application in quantitative morphological studies. 
The geometric and statistical theorems underlying these procedures, which might conveniently be called tensor biometrics, are summarized in Bookstein (1986). Appendix 4 of Bookstein et al. (1985) provides a simplified synopsis for the case of a single triangle of landmarks.

For shape-variations that are not too great, both the analysis and the interpretation of changes in landmark locations may proceed by considering the landmarks, three at a time, in a set of triangles distributed over the form to be studied. (In the example here, we refer to six triangles: ABC, ABD, ABE, $\mathrm{ABF}, \mathrm{ACD}$ and $\mathrm{AEF}$.) For complete coverage of $N$ landmarks, there must be at least $N-2$ triangles in a configuration that is rigid. When this requirement is satisfied, it does not matter which triangles are studied, as the multivariate statistical analysis of any such set is the same to terms of first order in the variation of shape (Bookstein, 1986). Any mean differences, trends, or statistical components of shape to be found in the landmark data may be construed as deformations of their configuration and may be visualized by the biorthogonal diagrams of Bookstein (1978). Alternatively, any mean difference or trend may be interpreted by way of the conventional distance measures which change in the greatest and in the least ratio across the comparison. These distance measures can always be characterized as measurements across triangles of landmarks (not necessarily the same triangles used in the statistical analysis). That is, they are distances taken from one landmark to the weighted average position of two others. Where these distances cross inside the form, they will lie at an angle averaging $90^{\circ}$.

The multivariate methods necessary to analyze the shape of a single triangle of landmarks are well-known from other biometric applications. The morphometric analysis of the shape of the triangle $X Y Z$ is equivalent to the ordinary normal-model analysis of the single complex variable $(Z-X) /(Y-X)$. (The arithmetic is that of complex numbers.) This is, in turn, the same as the ordinary normal-model multivariate analysis of the pair of Cartesian coordinates assigned to landmark $Z$ in a Cartesian system for which landmark $X$ is always set to location $(0,0)$ and landmark $Y$ to $(1,0) ; X Y$ is referred to as the "baseline". The construction results in what we call the shape coordinates of the triangle (cf. pp. 230-232 of Bookstein et al., 1985). Either of these statistical analyses, properly interpreted, is independent of which of the three landmarks is considered to be $X$ in the geometry, which $Y$, and which $Z$. Permutation of the landmarks $X, Y$, and $Z$ results, to first order, in mere rotation and rescaling of empirical scatters of shape. However these roles are assigned, a sample of triangular shapes becomes a scatter of these complex numbers or coordinate pairs. They may be analyzed in that form by ordinary multivariate methods, such as matched or unmatched $T^{2}$ tests, multiple regressions, canonical correlation analyses upon exogenous variables, and the like. 
Many statistical findings take the form of a vector of coefficients for the coordinates in this shape space: for instance, a mean difference, or an allometric dependency of shape upon size. Any such finding, re-interpreted as a deformation of the triangle of landmarks, may be immediately characterized by its principal directions, the pair of distances across it which increase most, respectively, least quickly. This last step, the generation of new variables that represent the particular comparison under study, proceeds by the geometrical constructions explained in Bookstein (1986) or Bookstein et al. (1985). It is these interpretations that are independent of which landmark was chosen to play $X$, which $Y$, and which $Z$ in the construction of the shape coordinates.

Size information was deleted from the statistical analysis when we standardized all the triangular forms to the same baseline length. The missing information on size is restored when that baseline length is included as an additional variable in the multivariate procedures. Studies of allometry do better if size is restored instead in the form of the root-mean-square of the edgelengths of the triangle. This particular size-measure is approximately uncorrelated with shape on a convenient null hypothesis, and so permits a single $F$-test for the existence of allometry whenever the assumptions of that null model can be verified. The null hypothesis states that shapes could have been derived by independent identically distributed circular normal variation about fixed mean locations. The size measures for triangles is a special case of the measure serving this function for the general configuration of landmarks, to wit, the root-mean-squared distance of all the landmarks from their centroid, as computed case by case.

When the shape coordinates are multiplied by the length of the baseline, they become the two Cartesian coordinates of a vector of displacement: the separation of landmark $Z$ from landmark $X$ in a coordinate system for which the baseline vector $X Y$ is horizontal. In the course of studying triangle ABD of the Brizalina data set, we shall have occasion to restore size information in this manner.

This entire family of statistical methods is independent of the choice of a coordinate system for digitizing. It is independent, also, of any a priori selection of size and shape variables for analysis. Instead the findings are dependent upon the choice of landmarks, the locations of which are the sole information about form available, and upon one's a priori notion of biological homology.

3.3. Findings. In this application of the tensor biometric procedures, we shall have no need for analyses of more than three landmarks at a time. Hence, all our findings can be presented in scatter-plots and diagrams in the same plane as the pictures of the specimens.

Our shape analysis is summarized in the pattern of mean shapes presented by level in Fig. 3. This figure shows the apparent motions of landmarks C, D, E 
and $\mathrm{F}$ in a coordinate system in which the aperture, point $\mathrm{A}$, is permanently fixed at location $(0,0)$ and the proloculus, point $B$, is permanently fixed at $(1,0)$; only levels $1,2,4$ and 5 are indicated. For all triangles, the scatter of shape for level 3 overlaps scatters for all the other strata, so that its relationships to them could not be clarified using these six points. The mean position of each landmark in this registered coordinate system is drawn for the four remaining levels as a line of three vectors, in the order 5421 .
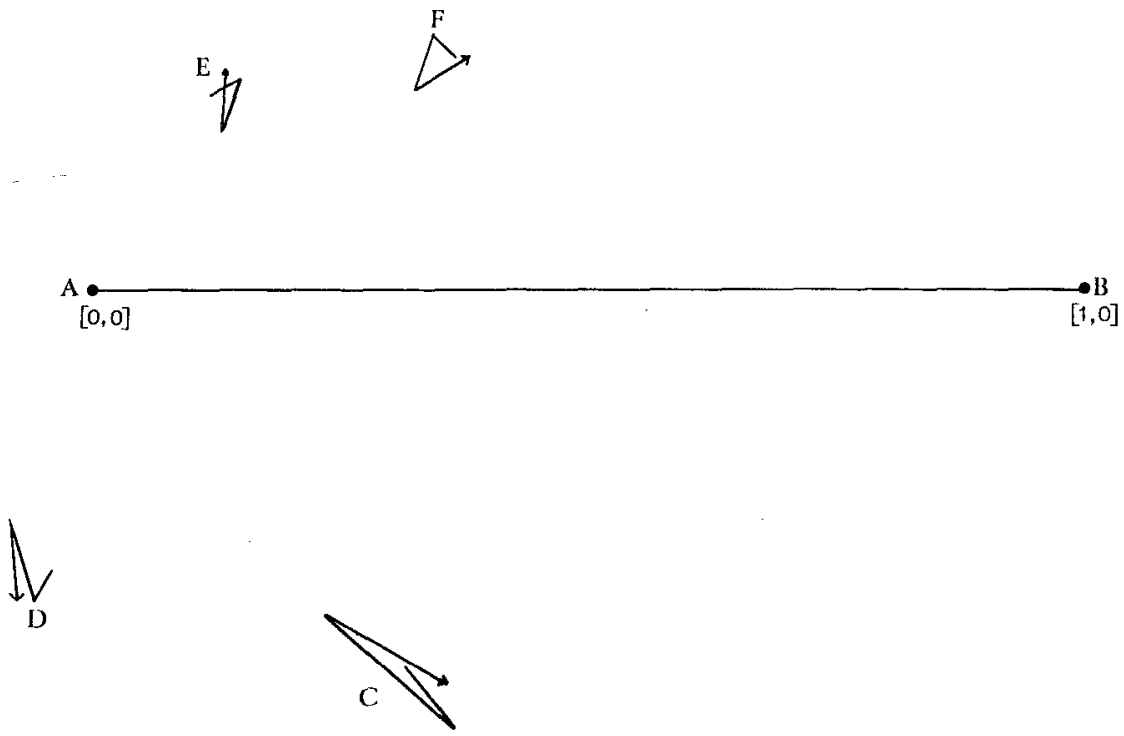

Figure 3. Trajectories of mean forms in shape-space for sampling levels $5 \rightarrow 4 \rightarrow 2 \rightarrow 1$ (in that order). The baseline is AB. The arrows are located at the highest level, namely, level 1.

Notice that, of the four landmarks considered to be "moving", the mean position for level 4 is close to that for level 1 for all but landmark F. Notice, too, that the change from level 5 to level 4 is not reliably associated with the path from level 4 to level 2 and back. Two landmarks, D and E, go off at a new angle to the old trajectory. Point $\mathrm{C}$ recapitulates part of the previous change; only point $\mathrm{F}$, traversing a triangle, returns to its relative position from level 5 . Notice that samples 1 and 5 hardly differ at all on any of the shape coordinates; their comparison involves mainly General Size, as we have already seen in the reinterpretation of Fig. 2.

Let us study these patterns of change in more detail. Figure 4 shows the locations of all 50 specimens in the space of shapes of triangle ABC, and outlines the scatters of subsamples 1,2 , and 4 within that scatter. The scatter, to be precise, represents the positions of the proximal limit of the final chamber in a coordinate system referred to the standard aperture-proloculus baseline. The 
specimens in the samples from levels 2 and 4 vary in opposite directions about a "core" representing the much lesser variation in level 1 . Figure 5 displays the mean shapes of triangle $\mathrm{ABC}$ for levels 2 and 4, the centroids of the corresponding sub-scatters in Fig. 4. Treated now as a deformation, instead of as the "motion" of one landmark, this change in shape has two principal directions of greatest relative effect. It is clear from the figure that length $\mathrm{AC}$ has decreased (from level 4 to level 2) relative to length $A B$, the "baseline" of the construction; but the geometry of alternate distance measures indicates that other ratios have changed considerably more. The simple ratio most sensitive to this particular shape change is the ratio $\mathrm{AC}: \mathrm{BC}$, the ratio of legs opposite the "hypotenuse" $\mathrm{AB}$ of this nearly right triangle.

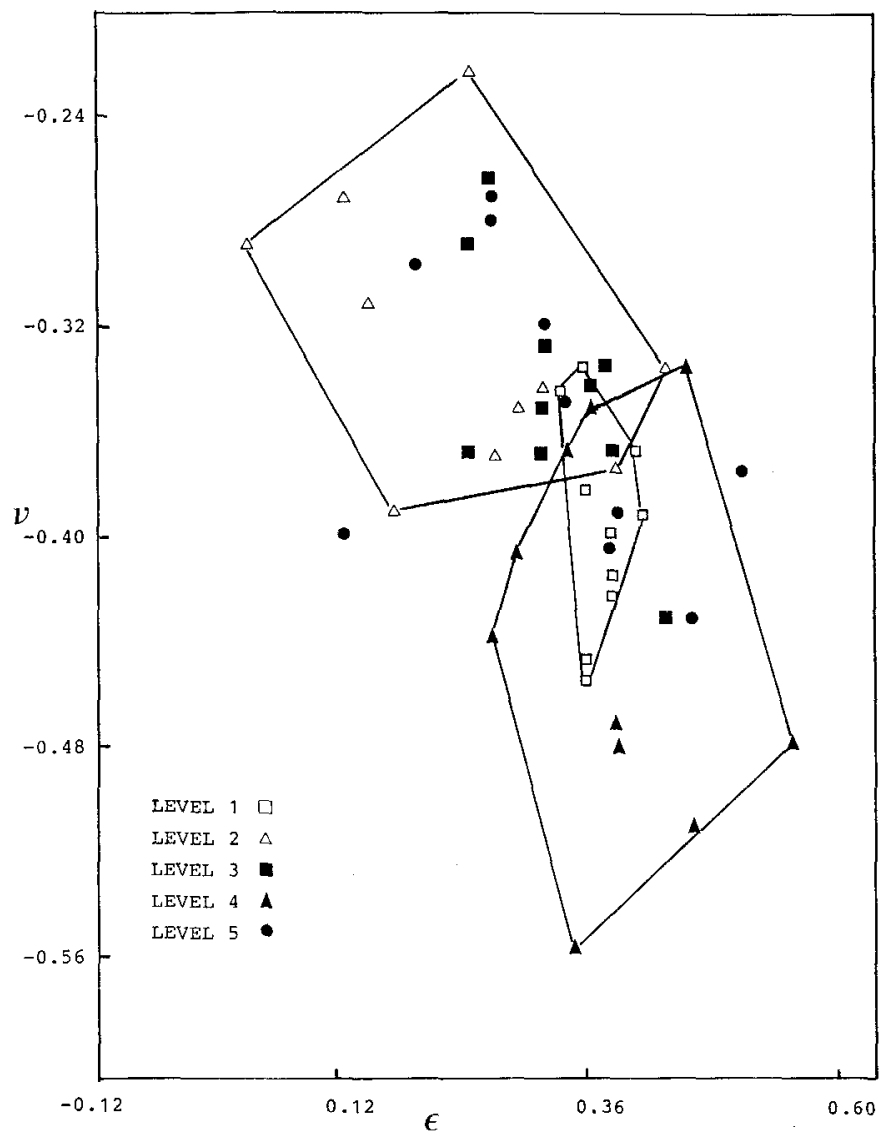

Figure 4. Location of 50 specimens in the space of shapes of triangle $\mathrm{ABC}$. The convex hulls of the scatters for samples 1,2 , and 4 are indicated. The respective levels are shown by means of symbols. The labelling of the axes follows the terminology of Bookstein et al. (1985). The abscissa $\varepsilon$ is denoted $\eta_{1}$ in Bookstein (1986), and the ordinate $v$ corresponds there to $\eta_{2}$. 
The same Fig. 5 indicates the relationship between the mean forms of triangle $\mathrm{ABF}$ in the two groups; the underlying scatters are presented in Fig. 6. In spite of the much greater overlap in this aspect of shape space, we may draw descriptive conclusions. Firstly, the difference between levels in the relation of the penultimate chamber to the baseline appears not to be the same as the difference in the relation of the final chamber to the same baseline. In the passage from level 4 to level 2, point $\mathrm{F}$ is displaced primarily inward, towards the baseline, whereas point $\mathrm{C}$ is also displaced distally, along the baseline. Secondly, two aspects of the "breadth" of the foraminifer (the distance [CF]) have changed with respect to its "length" (the distance $[A B]$ ). Not only has the ratio of these quantities decreased by $32 \%$ between level 4 and level 2, but also the angle has altered between the directions along which these distances are measured. As can be seen in Fig. 3, at level 4, as in the case of level 1, "breadth" is nearly orthogonal to "length", but at level 2, these distances make an angle averaging $77^{\circ}$ instead.

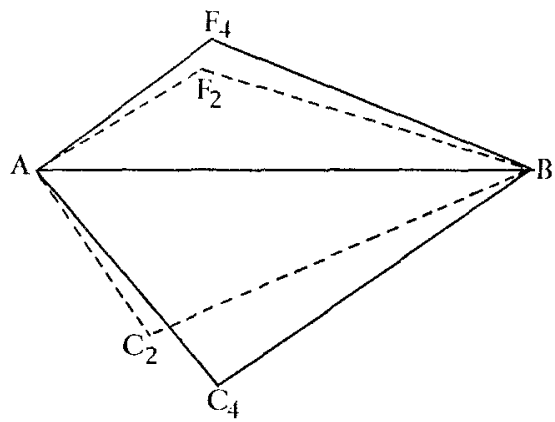

Figure 5. The mean shapes of triangle $\mathrm{ABC}$ for levels 2 and 4; that is, the centroids of the corresponding scatters in Fig. 4.

In addition to the position of the proximal ends $\mathrm{C}$ and $\mathrm{F}$ of the chambers, it is instructive to consider relations between the shapes of the chambers at these sampling levels. Figure 7 supplements the information in Fig. 5 by giving the apparent displacements of bulge-points $\mathrm{D}$ and $\mathrm{E}$ to the usual baseline; it also indicates the apparent displacements of points $\mathrm{C}$ and $\mathrm{F}$. Figure 8, computed separately, shows the relation between the mean shapes of the triangle ACD for levels 2 and 4 . The construction is by enlarging and rotating the triangle $\mathrm{AD} 2 \mathrm{C} 2$ so that the edge $\mathrm{AC} 2$ aligns with the edge $\mathrm{AC} 4$. The shape change from level 4 to level 2 is then shown by the straight arrow as the displacement from $\mathrm{D} 4$ to $\mathrm{D} 2{ }^{\prime}$, almost directly away from the edge AC. We see that the contrast is primarily one of relative height of the chamber, the ratio of this height to measure 3 of the standard set. This will be called the aspect-ratio below; it is larger in the sample from level 2 than that from level 4. The penultimate 


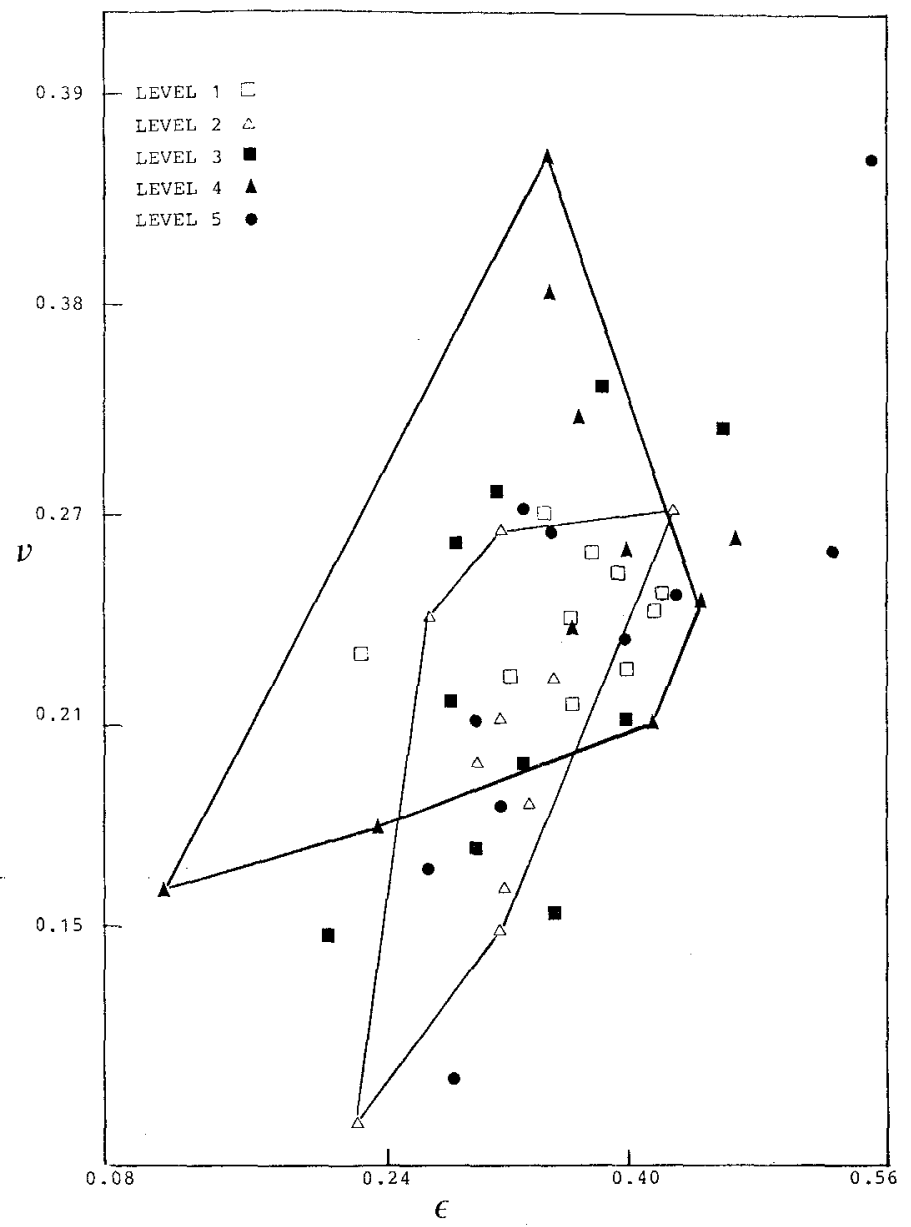

Figure 6. The scatters underlying the relationship between the mean forms of the triangle $\mathrm{ABF}$ in the samples from levels 2 and 4.

chamber shows no such difference. Apart from this shape change, it is tempting to interpret the reconfiguration of these landmarks between levels 4 and 2 as a relative reduction of the final chambers of the foraminifer (by two somewhat different factors) together with their rotation with respect to the body axis. Such a change could be simply explained as a displacement of point $B$, at the proloculus, a point which has hitherto been tacitly fixed through this discussion. If point $\mathrm{B}$ were to move somewhat with respect to the final chamber, then in a presentation with its position artificially fixed, points C, D, $\mathrm{E}$ and $\mathrm{F}$ would seem instead to move together and rotate clockwise about point A, just as we see in these data. Unfortunately, point B has not moved away from point $A$ at all (Fig. 9). When size information is restored to the baseline, so that we are now plotting the displacement vector from point $\mathrm{A}$ to 
points $\mathrm{C}, \mathrm{D}, \mathrm{E}$ and $\mathrm{F}$ according to a horizontal baseline, the two apparent changes of scale of the final chambers are found to be real, not merely relative.

We summarize the transition from level four to level two as follows. There has been a decrease in the ratio of "breadth" $[\mathrm{CF}]$ to "length" $[\mathrm{AB}]$, and a change in the angle between the segments underlying these distance-measures. The final chamber has somewhat decreased its diameter (more than has the penultimate chamber) but increased its own aspect ratio of height to base. The rotation between "breadth" and "length" is associated with the relative movement of the proximal end of this chamber distally along the body axis as well as toward it. The shape-change from level two to level one is just the opposite of this in all essentials.

3.4. Groups one and five. Groups one and five separate most in this study, but their contrast takes a quite different form from that between levels 2 and 1 or 4. We see in Fig. 3 that none of the shape differences between groups five and one is represented by large displacements on the plot of mean shapes. The longest of these displacements, that corresponding to triangle $A B C$, is not associated with any separation of scatters (Fig. 4), owing to the rather large scatter of shapes within group five.

One of the small differences is familiar. As Fig. 10 shows, using triangle $\mathrm{ACD}$, the aspect-ratio of the last chamber has increased by a few percent from level 5 to level 1. However, there is no apparent change in "breadth" [CF] nor in the angulation between "breadth" and "length", the other shape features of the transition from level 2 to level 4.

Nevertheless, this single shape effect, the change of relative position of the bulge of the last chamber, generates an almost perfect separation between the sampling levels in the shape of triangle ABD (Fig. 11). The long axes of the clusters in this figure are aligned in parallel with the segment from landmark $A$ to the mean position of landmark $\mathrm{C}$. That is, they parallel the direction of greatest indeterminacy in the location of point $\mathrm{D}$ (which was characterized, you will recall, as the point "farthest from the base of the chamber"). The coordinate of point $\mathrm{D}$ along this long axis carries no information at all about biological homology. We do not know "which point" we have located along the boundary curve, so to speak; we know only how far it is from the base of the chamber.

The separation between mean shapes for triangle ABD at levels 1 and 5 is approximately perpendicular to the baseline of the chamber; it lies in the direction of which the coordinate is meaningful in spite of the manner in which point $\mathrm{D}$ was characterized. We may interpret this coordinate, then, as if it were the height of the last chamber, analogous to measure 1 of the first set.

At landmark E, by contrast, the apparent displacement of the "top" of the bulge from level 1 to level 5 is directly along the direction of uncertainty of 


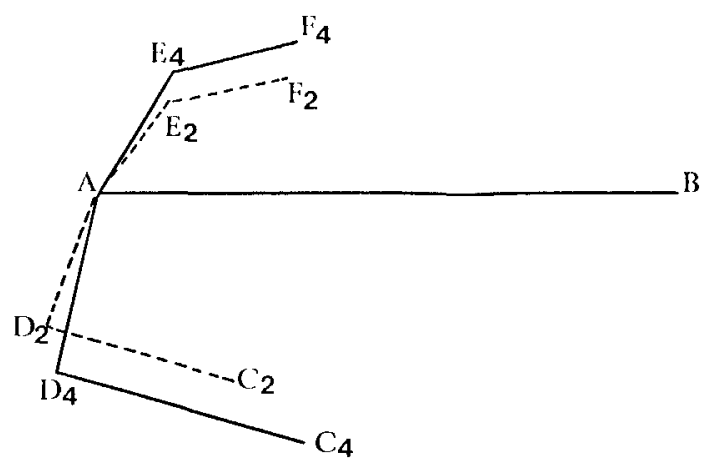

Figure 7. Augmentation of Fig. 5 to include apparent displacements of the bulgepoints $\mathrm{D}$ and $\mathrm{E}$, and points $\mathrm{C}$ and $\mathrm{F}$, to the usual baseline $\mathrm{AB}$.

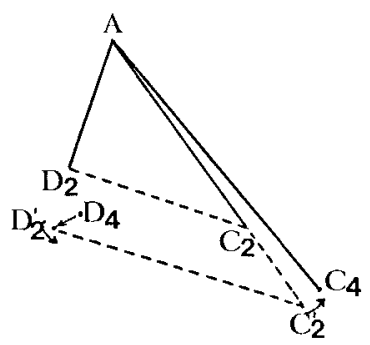

Figure 8. Relation between the mean shapes of the triangle ACD for levels 2 and 4 . The construction is as given in Appendix A2, steps 1 and 3, of Bookstein et al. (1985).

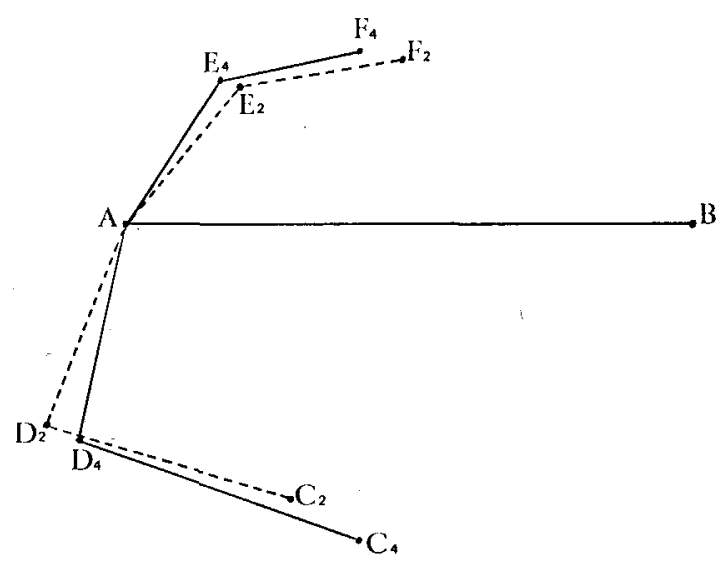

Figure 9. Mean positions of landmarks $D$ and $E$, and $C$ and $F$, when the size of the baseline is restored to the analysis. The change in shape from level 4 to level 2 is not explained by relative motion of the point $B$ (the proloculus). 


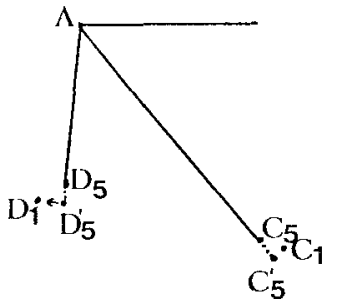

Figure 10. Triangle ACD at levels 1 and 5. The aspect-ratio of the last chamber increases from level 5 to level 1 . There is no change in "breadth", [CF], nor in the angulation between "breadth" and "length".

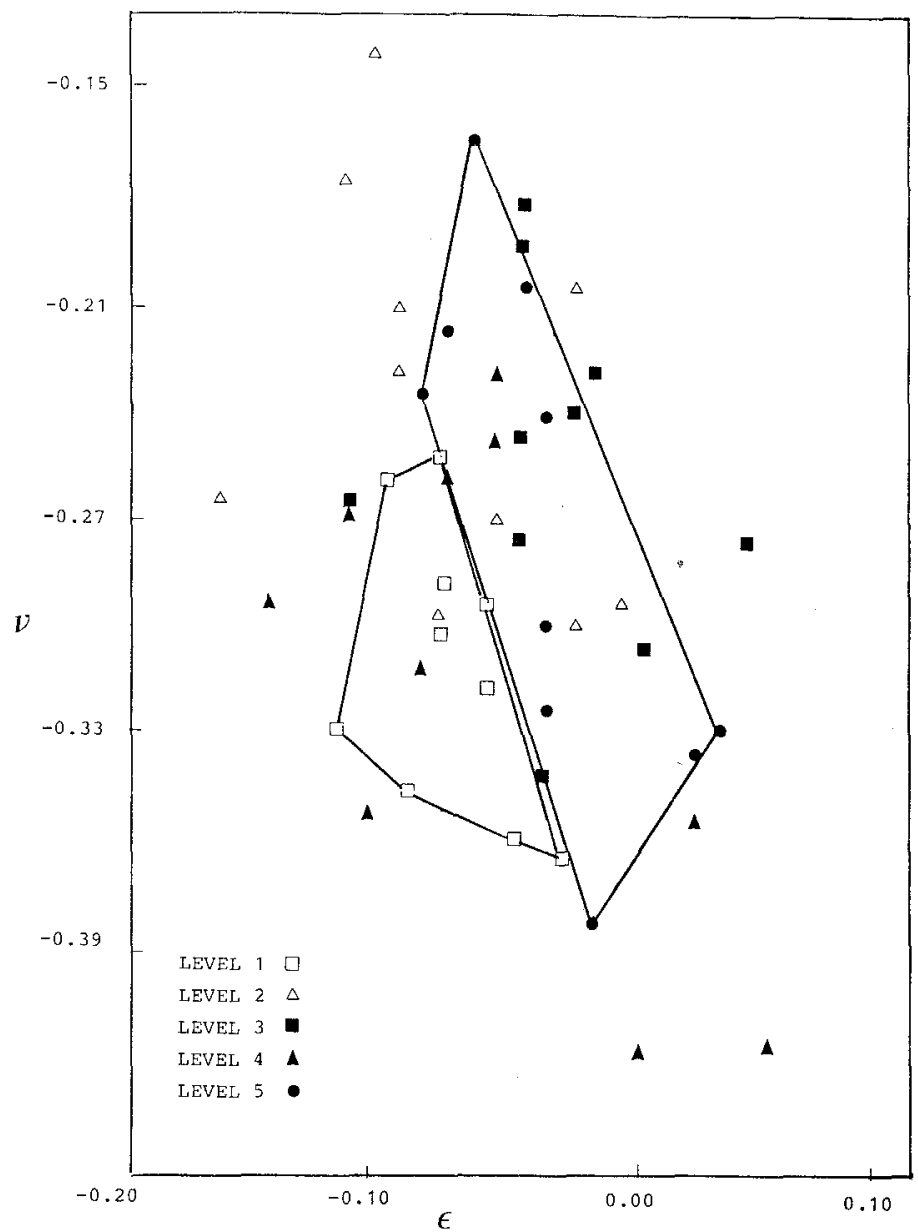

Figure 11. Scatter for triangle ABD. The long axes of the clusters parallel the segment from landmark $A$ to the mean position of landmark $C$. 
location of point $\mathrm{E}$, so that there is no need to invent a biological interpretation for this displacement. We may conclude only that the penultimate chamber does not bulge out further in the material from level 1.

In addition to the mean difference in shape between levels 5 and 1, there is a considerable difference in average size. In Fig. 12, we take advantage of this factor by reverting from the shape coordinates to correctly scaled vectors of relative displacement. There is no resulting separation, the size differences being manifested in the nesting of sample 1 (the youngest) within the scatter of sample 5 (the oldest). Like the shape coordinate underlying it, the mean difference of this vector, which is in the direction of "height" of the final chamber between the levels, lies perpendicular to the average direction of uncertainty of location of point $D$.

4. Discussion. We may extract three conclusions from this demonstration: about Brizalina (Section 4.1), about morphometric data in general (Section 4.2) and about morphometric statistical method (Section 4.3).

4.1. Brizalina. Figure 3 suggests that there is randomness in the redirection of changes between levels of Brizalina, an impression borne out by the detailed analysis immediately above. The difference between levels 1 and 5 bears no particular relationship to the difference between levels 2 and 4 . We saw this indirectly in the canonical variate plot, where the separation between centroids of groups one and five made an angle close to $90^{\circ}$ with the separation between the centroids of groups two and four. We saw it more clearly in the analysis of triangles of landmarks, where, of three shape descriptors sensitive to the mean change in form from level 4 to level 2, only one was found to characterize the transition from level 5 to level 1, while the others, aspects of the geometry of "length" and "breadth", were replaced by a criterion of global size difference. Furthermore, the canonical analysis exaggerates the separation between groups one and four, which are almost identical in mean shape.

We should like to propose the hypothesis that these Brizalina are "wandering" in phenotypic space rather than following any sort of controlled gradient or trend; a random walk without drift, as it were, so that, for instance, after the first return of the organism to the form of level 5, it sets off in an unrelated direction. Perhaps this is an example of evolutionary stasis (Eldridge and Gould, 1972; Reyment, 1983). Size has changed substantially over this series; the aspect ratio of the last chamber has increased over the same span of values, but other aspects of shape-change occurred only once, such as the rotation of angle between "breadth" and "length".

Whenever new directions in shape-space are randomly introduced, sample after sample, as for these foraminifers, no single canonical analysis nor other application of biologging (Reyment, 1980) is likely to be capable of adequately 


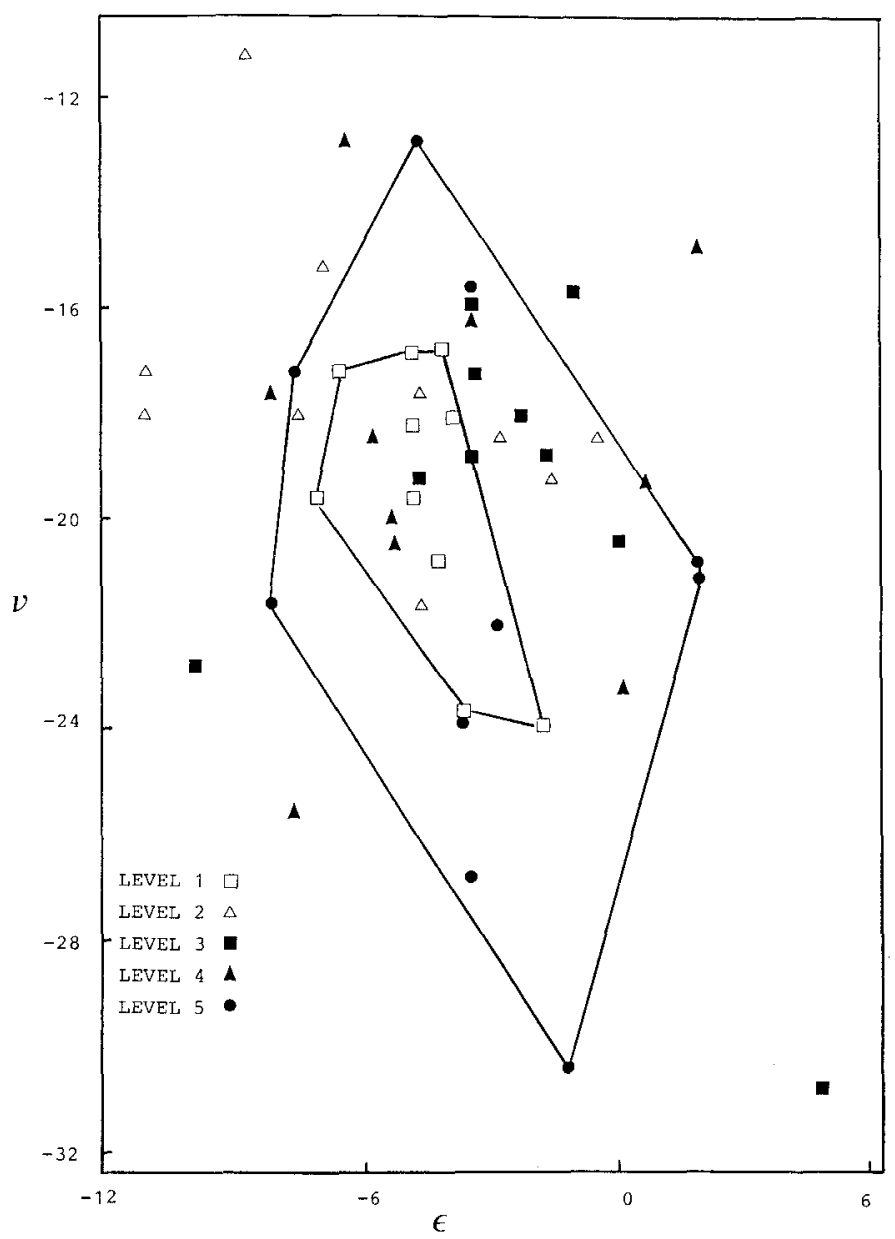

Figure 12. Triangle ABD, with size included. Here the scatter for the points of level 1 lies within that for level 5.

following the trajectory. To illustrate this concept, we shall consider a homely analogy. Think of an ordinary paperclip which has been partially unbent into a non-planar form bearing three successive right angles in three orthogonal planes. A canonical analysis will find the diagonal of this construction, the "axis" from one end to the other of the wire. This direction is, however, not meaningful within morphological space. It is an average of the three random directions of morphological variation adopted at the three different "steps" of the folding paperclip, and bears no implications about the subspace for subsequent changes.

A great deal of the argument about tempo and mode of evolution is vitiated by poverty of variables. When a sufficiently rich representation of morphologi- 
cal space is available, a representation easiest to generate by means of landmarks, it seems to us quite likely that at every epoch something new is being attempted, some old experiment abandoned, and some trend continued. The artificial restriction of the descriptor space to a number of variables fewer than the number of samples conceals the possibility of this renewal from our biometric purview. Once again using the analogy of the paperclip, we see that every bend of the wire, every succession of levels, is a movement in morphospace potentially unrelated to the subspace of the full previous history.

4.2. Morphometric data. A set of six landmarks, such as we collected here, bears nine statistical degrees of freedom: eight for shape (for instance, the eight shape coordinates for triangles $\mathrm{ABC}, \mathrm{ABD}, \mathrm{ABE}, \mathrm{ABF}$ ) together with a single additional dimension for size, to be measured only once in any sensible fashion. In practice, particular combinations of these degrees of freedom have intuitive meanings all their own. For instance, we discovered the apparent rotation of "breadth" with respect to "length" between levels while not measuring length, breadth, nor this angle in any explicit way. Ordinary ratios, which could have been measured, but were not, were instead generated in the course of the data analysis as natural interpretations of the coordinate-free findings. In this way, we hit upon the aspect ratio involving points $A, C$ and $D$ of the ultimate chamber, but also upon the unfamiliar ratio $\mathrm{AC}: \mathrm{BC}$ which proved the most sensitive description of the difference between level 4, or level 1, and level 2 .

By comparison, a set of distance measures, such as the five that were treated in the canonical variate analysis, does not seem to expand into such complete coverage of the form. The computed contrasts of variables which are the canonical variates of Table III, for instance, seem interpretable only in those tabulations, as computed contrasts bearing certain properties of optimality. Nowhere in the canonical analysis, for instance, do we learn that the first canonical variate is almost exactly a combination of scale and shape information about the position of one point, the bulge of the last chamber, with relation to the aperture and the point at the proloculus. Once scale is divided out, along with the rest of the within-groups matrix, the canonical coefficients obscure the fact that covariance in size remains substantial. Nor can we learn anything from the conventional character set about measures such as the angle between "breadth" and "length", as the information regarding that angle was simply not gathered. (A wiser choice of variables is, of course, always possible and could lead to a more informative outcome-see Section 4.3.)

4.3. Morphometric methods. It is possible to ensure the effective coverage of landmarks by a conventional character set of distances if the distances are measured between landmarks in a determined or overdetermined network (Strauss and Bookstein, 1982; Skalak et al., 1982; Section 3.2 of Bookstein et $a l ., 1985)$. In this case, it can be shown that the space spanned by the distance 
variables, i.e. the statistical space accessed by multivariate methods, is equivalent to the statistical spaces of shape and size underlying the analysis of triangles as exemplified in this essay. (The theorems supporting this assertion are proved in Sections 2 and 3 of Bookstein, 1986.) Nevertheless, after statistical computations are completed, the problem of interpretation remains. It is easy to construct simple geometric operations (deformation of a region by a tumor, or rigid motion of one set of landmarks with respect to another set) which are very well hidden by their explicit recording as changes of log-distances.

The clearest way of interpreting findings from a multivariate analysis of distances between landmarks is by reverting to some set of triangles, as demonstrated here, then constructing the principal directions corresponding to the tensor interpretation of the coefficients computed: the distances that change most and least quickly. Theorem 2 of Bookstein (1986) warrants that such triangles can always be found. In other words, for any multivariate trend or contrast involving distances measured among however many landmarks, the interpretation of multivariate analysis by means of triangles is sufficient. In our view, the interpretation by triangles or other geometric representations of the data is, as well, necessary.

In the present example, the choice of baseline was straightforward on biological grounds. Point B (proloculus) is laid down once and for all in embryo, and point A (aperture) remains homologous over development even as growth is repeatedly renaming $(C, D)$ pairs as $(F, E)$ pairs. In other applications of the shape coordinates, the biometrician has more freedom in choosing baselines. The selection of $\mathrm{AB}$ here is consistent with the general advice in Bookstein (1986) suggesting the use of the longest diameter of the form. The theorems there guarantee that the appropriate (tensor) interpretation of multivariate statistical analyses is approximately invariant under change of baseline.

The form of Brizalina is assembled out of parts, and the description reported here describes the parts (chambers) and their assembly (the vertex angle). In general, the task of describing the findings of a shape coordinate analysis is more fluid than this. A general review of techniques may be found in Chapter VII of Bookstein (1990). Typically one turns to one or another hierarchy of invariant descriptors of global aspects of these changes. Two possibilities are by polynomials of increasing degree (basically an idea of Peter Sneath's-see Bookstein and Sampson, 1987; 1989) and by eigenfunctions of "bending energy" of varying geometric scale (Bookstein, 1989a; 1989b). These two decompositions share their first term, the component of uniform shear, about which a small literature of estimation is growing on its own (Rohlf and Slice, submitted; Goodall, submitted; Mardia, 1989).

Preparation of this paper was supported in part by USPHS grant GM-37521 to Fred L. Bookstein and by Swedish Natural Science Research Council grants G-GF 2320-134 and G-GU 2320-129 to R. A. Reyment. 


\section{LITERATURE}

Bookstein, F. L. 1978. The Measurement of Biological Shape and Shape Change. Lect. Notes Biomath., Vol. 24.

_. 1982. "Foundations of Morphometrics." Ann. Rev. Ecol. Systematics 13, 451-470.

. 1986. "Size and Shape Spaces for Landmark Data in Two Dimensions." Statist. Sci. 1, $181-242$.

— 1989a. "Comment on D. G. Kendall A Survey of the Statistical Theory of Shape." Statist. Sci., 4, 99-105.

1989b. "Principal Warps: Thin-Plate Splines and the Decomposition of Deformations." IEEE Trans. Patt. Anal. Mach. Intel., 11, 567-585.

- 1990. Morphometric Tools for Landmark Data. Cambridge University Press, accepted for publication.

_ and P. Sampson. 1987. Statistical Models for Geometric Components of Shape Change." Proceedings of the Section on Statistical Graphics: Annual Meeting of the American Statistical Association, pp. 18-27.

and 1989. "Statistical Models for Geometric Components of Shape Change." $J$. Am. Statist. Assoc., submitted.

- B. B. Chernoff, R. Elder, J. Humphries, G. Smith and R. Strauss. 1985. Morphometrics in Evolutionary Biology. Special Publication No. 15. Academy of Natural Sciences of Philadelphia.

Brower, J. C. and J. Veinus. 1978. "Multivariate Analysis of Allometry Using Point Coordinates." J. Paleontol. 52, 1037-1053.

Eldredge, N. and S. J. Gould. 1972. "Punctuated Equilibria: An Alternative to Phyletic Gradualism." In Models in Paleobiology, T. Schopf (Ed.), pp. 82-115. San Francisco: Freeman Cooper.

Goodall, C. R. 1983. "The Statistical Analysis of Growth in Two Dimensions." Doctoral Dissertation, Department of Statistics, Harvard University.

- 1989. "WLS Estimators and Tests for Shape Differences in Landmark Data." J. R. Statist. Soc., submitted.

Hopkins, J. W. 1966. "Some Considerations in Multivariate Allometry." Biometrics 22, 747-760.

Mardia, K. V. and I. L. Dryden. 1989. "The Statistical Analysis of Shape Data." Adv. appl. Probability, in press.

Reyment, R. A. 1980. Morphometric Methods in Biostratigraphy. London: Academic Press.

—. 1982. "Phenotypic Evolution in a Cretaceous Foraminifer." Evolution 36, 1182-1199. 1983. "Phenotypic Evolution in Microfossils." Evol. Biol. 16, 209-254.

, R. E. Blackith and N. A. Campbell. 1984. Multivariate Morphometrics (2nd edn). London: Academic Press.

Rohlf, F. J. and D. Slice. 1989. “Methods for Comparison of Sets of Landmarks.” Systematic Zool., submitted.

Siegel, A. F. and R. H. Benson. 1982. "A Robust Comparison of Biological Shapes." Biometrics 38, 341-350.

Skalak, R., G. Dasgupta, M. Moss, E. Otten, P. Dullemeijer and H. Vilmann. 1982. "Analytical Description of Growth." J. theor. Biol. 94, 555-577.

Sneath, P. H. A. 1967. "Trend-Surface Analysis of Transformation Grids." J. Zool. 151, 65-122.

Strauss, R. F. and F. L. Bookstein. 1982. "The Truss: Body Form Reconstructions in Morphometrics." Systematic Zool. 31, 113-135.

Thompson, D'A. W. 1961. On Growth and Form (abridged edn), J. T. Bonner (Ed.). Cambridge University Press. 